\title{
Virtual Instrumentation Applied to Identifying Parameters of Solar Radiation and Ambient Temperature Using Autoregressive Modeling with Exogenous Inputs
}

\author{
A.J. Aristizábal \\ Departamento de Ingeniería, Universidad de Bogotá Jorge Tadeo Lozano, Bogotá, Colombia
}

\begin{abstract}
This work describes a mathematical model developed to identify solar radiation and ambient temperature patterns in Bogota, Colombia, using autoregressive modeling with exogenous inputs (ARX). The solar radiation data and temperature used in the model were taken from the meteorological stations of Universidad de Bogotá Jorge Tadeo Lozano and Instituto de Hidrología, Meteorología y Estudios Ambientales - IDEAM from Colombia; for a period of 5 years (2011-2015). The model (ARX) was implemented using the LabVIEW graphical programming language, and allow identification of solar radiation parameters using techniques MSE. After applying the ARX model, the results showed that the correlation between the solar radiation measured by the monitoring station and evaluated by the algorithm was 86.64\%; while for the temperature was $76.15 \%$ for the same period. This type of parameter identification is for application in the field of photovoltaic solar energy as it might be possible to identify future capabilities of generating electricity with solar panels.
\end{abstract}

Keywords: Virtual instrumentation, ARX model, radiation and temperature.

\section{Introduction}

Extended research is devoted to estimate the photovoltaic infield conversion efficiency as a prerequisite for accurate design of feasible and affordable systems; literature outlines that the main factors affecting the conversion efficiency are: the PV type/materials, the amount of incident solar irradiance and the operating temperature [1,2]. These factors are further depending on specific features of the implementation location (geographical coordinates, climatic profile) and on parameters such as the ambient and PV module temperature, variation with wind, etc [3].

The energy and environment issues have become increasingly serious with the development of society, solar energy, as a kind of renewable energy, has a good application prospect. However, solar power generation has still some problems as follows: the conversion efficiency of solar cells is lower, and the output power of photovoltaic (PV) array has great relationship with irradiation and temperature $[4,5,6]$.

Many studies have been devoted to develop different non-linear electric models used to describe the characteristics of the PV modules and the effect on module performance of temperature, radiation intensity and other parameters under non-standard conditions [7-22].

Time series models have been obtained from econometric advances, which is a science that grows from the statistics of data obtained [23]. These models are applicable to a diversity of cases in which information is arranged accordingly to analyze the information system's behaviour.

This paper describes and applies a regression ARX model to the database of solar radiation and ambient temperature data recorded in the city of Bogotá, from 2011 to 2015.

\section{Theoretical Analysis}

\subsection{Application of ARX and MSE Models to Irradiance and Temperature Patterns}

\subsubsection{Model ARX}

The models presented in this paper follow the structure of model ARX (autoregressive with exogenous inputs) modified for application of time series $[24,25,26]$. This model is built with (Input-output measured system data) data measured of input and output of a system, in which the behaviour of the system can be represented through two vectors called input regression size $n+1$ and output regression size $n$. The coefficients aj and bj are parameters to be estimated applying any kind of numeric analysis technique, for example least squares. The term $\mathrm{e}(\mathrm{k})$ is associated to disturbances of the systems, not represented by the behaviour of an equation but by a numerical value $[24,25]$.

$$
y(k)=\sum_{j=1}^{n} a_{j} y(k-j)+\sum_{j=0}^{n} b_{j} u(k-j)+e(k)
$$


Now, as this is a model with exogenous inputs, we take from (1) and join the terms of the second series (bj with its respective regression) to reduce it to a constant $\gamma$, retaining its structure to represent mathematically time series as shown in (2). In this way, the new model is called model AR, since it will just depend on an output regression vector plus a constant. [24,26].

$$
y(k)=\sum_{j=1}^{n} a_{j} y(k-j)+\gamma
$$

In order to be able to do one or several predictions with this model, we should shift in (2). For this shift, it is required to define the number of predictions required $i$, being $\mathrm{i}=1,2,3, \ldots \mathrm{N}$, where $\mathrm{N}$ is the maximum number of predictions required to obtain the expression that represents the model of prediction $y(k+i)$. After doing this shifting, in a general manner, it remains as it is shown in (3).

$$
y(k+i)=\sum_{j=1}^{n} a_{j} y(k-j+i)+\gamma \quad i=1,2, \ldots, N
$$

\subsubsection{Model MSE}

This is a numerical analysis technique developed by Karl Friedrich Gauss [27], which serves to optimise the square error $\mathrm{E}(\mathrm{k}) 2$ among the data observed and the data estimated. The summation represented in (4) represents all the square errors to model for in the data $\mathrm{k}=1,2, \ldots \mathrm{N} k=1,2, \ldots, N$. This function will help us to calculate the coefficients of the polynomials of a transfer function that better represents the data observed [24,27].

$$
I(N)=\sum_{k=1}^{N} e(k)^{2}
$$

Taking from (2) and replacing in (4) we obtain the expression (5).

$$
I(N)=\sum_{k=1}^{N}\left(\hat{y}(k)-\left(\sum_{j=1}^{n} a_{j} y(k-j)+\gamma\right)\right)^{2}
$$

In order to solve (5) we should do the partial derivatives with respect to all coefficients of the transfer function to obtain a lineal equations system, which, in this case, it is represented by the multiplication of regression vectors $\mathrm{c}(\mathrm{n}+1), 1$ y $\mathrm{f}(\mathrm{n}+2), 1$ in (6), obtaining an equations matrix for each model in an instant $\mathrm{k}(7)$, then, at the end there is a summation of equation matrices given by (8) which represents the system with a transfer function of order $n$.

$$
\begin{gathered}
c(k, n)=\left[\begin{array}{c}
y(k-1) \\
y(k-2) \\
\vdots \\
y(k-n) \\
-1
\end{array}\right] \quad f(k, n)=\left[\begin{array}{llllll}
y(k-1) & y(k-2) & \cdots & y(k-n) & -1 & -y(k)
\end{array}\right] \\
M_{n+1, n+2}(k, n)=c(k, n) * f(k, n) \\
M T_{n}=\sum_{k=n}^{N} M(k, n)
\end{gathered}
$$

Now with the total matrix of linear equations, it is necessary to apply a method of reduction to find the coefficients, in this case, we use the Gauss-Jordan method and the result of the coefficients is given by (9), where $\mathrm{r} 1, \mathrm{r} 2, \ldots \mathrm{rn}+1$ are the result of the reduction. 


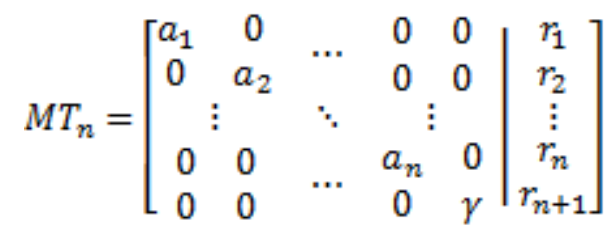

Once the coefficients of the polynomial regression and the constant are obtained the model can be evaluated or make predictions with (3).

\subsection{Monitoring System}

The measurement system was implemented by Aristizábal et al. $(2008,2011)$ and extensively described in other articles [28,29]. This is installed at Universidad de Bogotá Jorge Tadeo Lozano.

The monitoring system was developed using the Virtual Instrumentation concept [30]. Thus, compact DAQ devices of National Instruments Company were used as hardware and the LabVIEW (Laboratory Virtual Instrument Engineering Workbench) package [31] was used as software. The system includes sensors and transducers for measuring the parameters required to measure the solar radiation and ambient temperature.

Technical specifications of the sensors and acquisition system are as follow:

- Solar Irradiance sensor: Piranometer Kipp \& Zonen SP-LITE ( Response Time <1s, Sensitivity of $72 \mu \mathrm{V} / \mathrm{W} / \mathrm{m} 2$, spectral range $0.4-1.1 \mu \mathrm{m}$, stability $< \pm 2 \%$ /year, non linearity $<1 \%$ up to $1000 \mathrm{~W} / \mathrm{m} 2$ ).

- Temperature sensor: $10 \mathrm{k}$ Thermistor (Time Constant of $2.5 \mathrm{~s}$, dissipation Constant of $8 \mathrm{~mW} /{ }^{\circ} \mathrm{C}$, tolerance 0 $\left.70^{\circ} \mathrm{C}\right)$

- Acquisition System: NI cDAQ-9174 system with the following conditioning input modules:

- $\quad$ NI 9205: 32-Ch $\pm 200 \mathrm{mV}$ to $\pm 10 \mathrm{~V}, 16$-Bit, $250 \mathrm{kS} / \mathrm{s}$ Analog Input Module.

- $\quad$ NI 9225: 300 Vrms, Simultaneous Analog Input, 50 kS/s, 3 Ch Module.

\subsection{Virtual Instruments}

The signal measuring and the acquisition, processing, storing and reporting of the whole data, were achieved through a virtual instrument (VI) developed with the help of the LabVIEW package. The data were stored in the computer hard disk in a universal format, allowing their subsequent processing with LabVIEW or any other known electronic spreadsheet.

The monitoring of the whole system was performed with several sub-VI's which are integrated to the main VI through the source code contained in the block diagram. The numerical data and graphics generated with each sub-VI can be visualized in the computer screen by activating a window displayed in the upper side of the frontal panel of the main VI [31].

Fig. 1 shows the front panel of the VI displaying numerical values of solar radiation and ambient temperature.

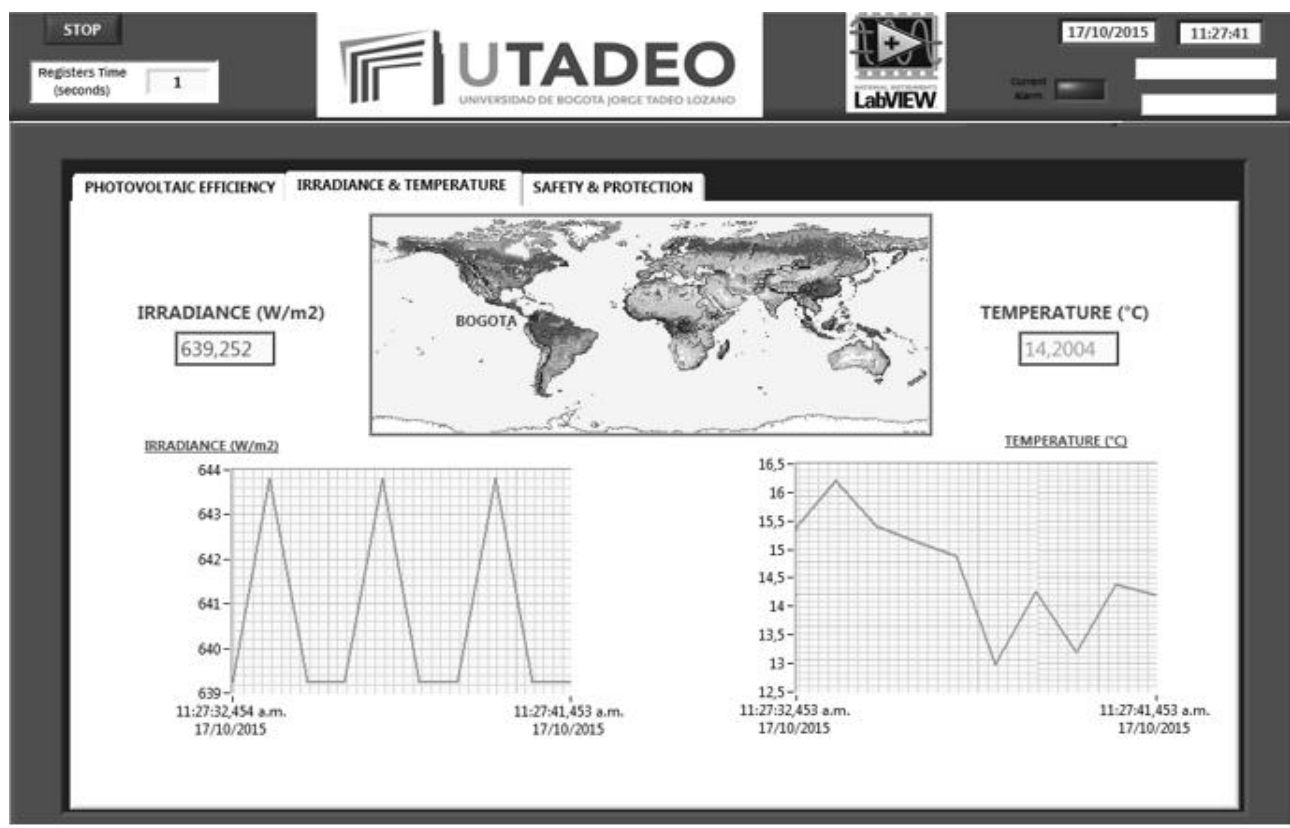

Fig. 1. Front panel of the VI developed for monitoring the solar radiation and ambient temperature. 


\section{Results and discussion}

Solar Radiation and ambient temperature data were obtained from the meteorological station at Universidad de Bogotá Jorge Tadeo Lozano for 2015 and from IDEAM database for 2011 - 2014.

\subsection{Autoregressive model implemented in LabVIEW}

The algorithm with exogenous inputs (ARX) was implemented in LabVIEW and the operating process is as follows [24]: to verify.

Data to be entered by the user are: Vector of input data, vector of output data, and numbers of models

Step 1: All the local variables are initiated and the size or number of input and output vector data is calculated.

Step 2: Two vectors with entry and output data corresponding to the current order are organized, one size $(n+$ 1) and the other $(n+2)$, the same way as the partial derivatives are represented to identify, through MSE, a system of one entry and one output (SISO) (ec. (6)). These two vectors are multiplied to obtain a matrix of $([n+1] x[n+2])$ that are added up to the matrix of the previous iteration. This step is repeated until all entry and output data are shifted.

Step 3: From the resulting matrix of the previous step, we proceed to make a reduction through the GAUSSJORDAN method. Obtaining the coefficients of a difference equation, we can represent them as polynomials of a transfer function corresponding to the estimated model (ec. 9).

Step 4: With the coefficients of the difference equation, we move on evaluate the model with input data to obtain an estimated response. This response is compared to the real data through a correlation.

Step 5: Evaluating the correlation obtained, the order of the model that is closest to a correlation of $100 \%$ is stored. After evaluating the correlation, the order is increased and we repeat step 2, to evaluate another model of a higher order. This is done until all the orders proposed by the user, at the beginning, are checked.

Step 6: Once all the models have been evaluated, the order that has the best correlation can be obtained. We reevaluate the coefficients for this order and in this way, find the transfer function that best represents the data.

The structure of the model used was the following [10]:

$$
y(k)=\zeta-\left(a_{1} y(k-1)+a_{2} y(k-2)+\cdots+a_{n} y(k-n)\right)
$$

\subsubsection{Solar Radiation}

To obtain the most appropriate model, we entered the 60 irradiance data of Bogotá city, which are equivalent to five years (one data per month), and through MSE, we found the order 32 and the coefficients are represented in the Table 1 . With $\zeta=1.6712$, the correlation obtained was $86.64 \%$.

\begin{tabular}{|l|l|l|l|l|l|l|l|l|l|}
\hline a1 & 0,2456 & a2 & 0,1230 & a3 & $-0,0276$ & a4 & 0,3421 & a5 & 0,3315 \\
\hline a6 & 0,2634 & a7 & $-0,1982$ & a8 & $-0,1741$ & a9 & 0,4231 & a10 & $-0,3564$ \\
\hline a11 & 0,0103 & a12 & $-0,1122$ & a13 & 0,0212 & a14 & $-0,3657$ & a15 & $-0,2454$ \\
\hline a16 & 0,0890 & a17 & $-0,1783$ & a18 & 0,2867 & a19 & $-0,0871$ & a20 & 0,4287 \\
\hline a21 & $-0,3177$ & a22 & 0,1163 & a23 & $-0,4128$ & a24 & $-0,3769$ & a25 & 0,3241 \\
\hline a26 & 0,0370 & a27 & 0,1321 & a28 & $-0,4166$ & a29 & 0,3862 & a30 & 0,2169 \\
\hline a31 & $-0,0132$ & a32 & 0,3538 & & & & & & \\
\hline
\end{tabular}

Table 1. Coefficients obtained for the model of solar radiation of order 32.

\subsubsection{Temperature}

To obtain the most appropriate model, we entered the 60 irradiance data of Bogotá city, which are equivalent to five years, and through MSE, we found the model.

The model obtained was of order 12 and the coefficients are represented in Table 2.

With $\zeta=18.5423$, the correlation obtained was $76.15 \%$.

\begin{tabular}{|l|l|l|l|l|l|l|l|l|l|}
\hline a1 & 0,6743 & a2 & $-0,4321$ & a3 & 0,1187 & a4 & $-0,0046$ & a5 & 0,5341 \\
\hline a6 & 0,3731 & a7 & $-0,1589$ & a8 & 0,1351 & a9 & $-0,5482$ & a10 & 0,0865 \\
\hline a11 & $-0,4231$ & a12 & $-0,2461$ & & & & & & \\
\hline
\end{tabular}

Table 2. Coefficients obtained for the temperature model of order 12.

In Figs. 2 and 3 we can observe the results of models that were applied for the solar radiation as well as for the temperature using the algorithm in LabVIEW. 


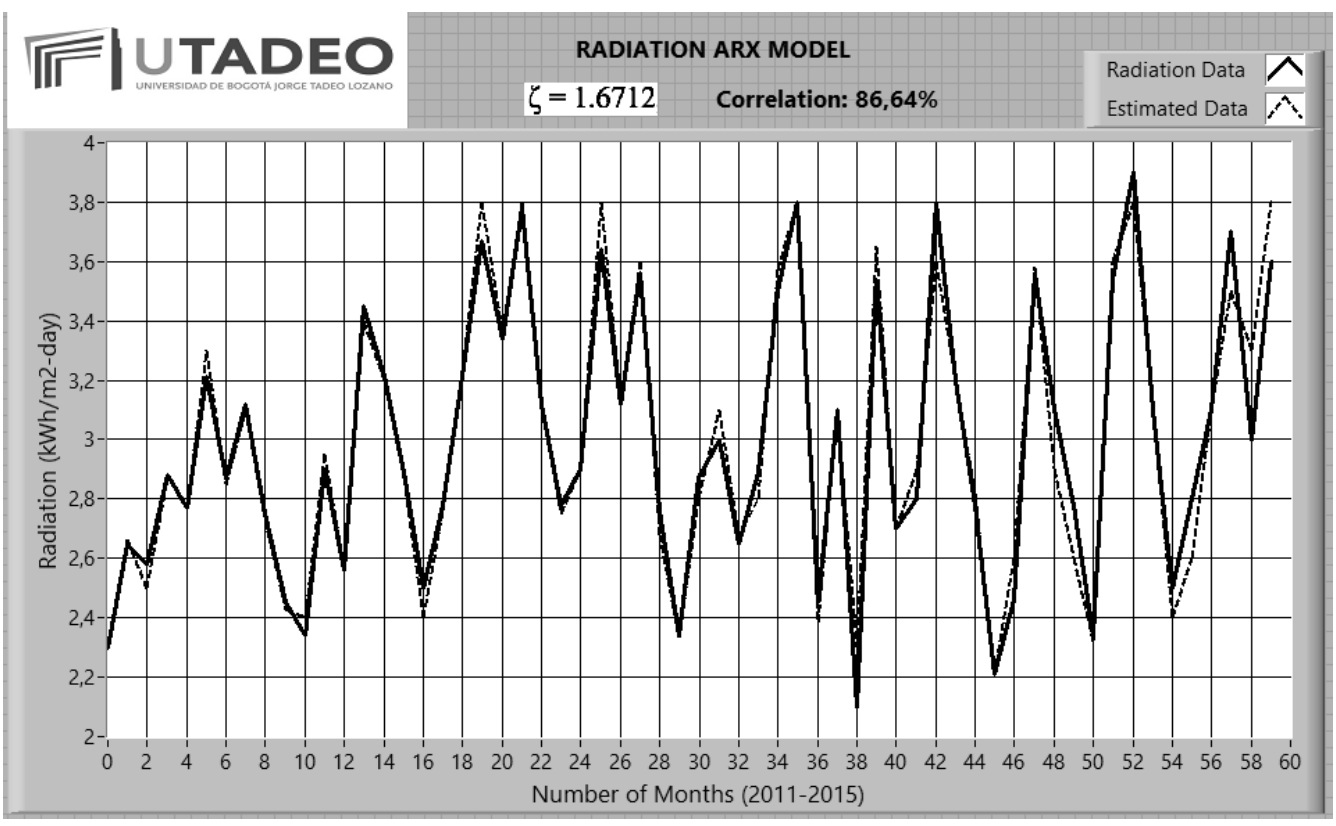

Fig. 2. Behavior for real radiation and the model estimated.

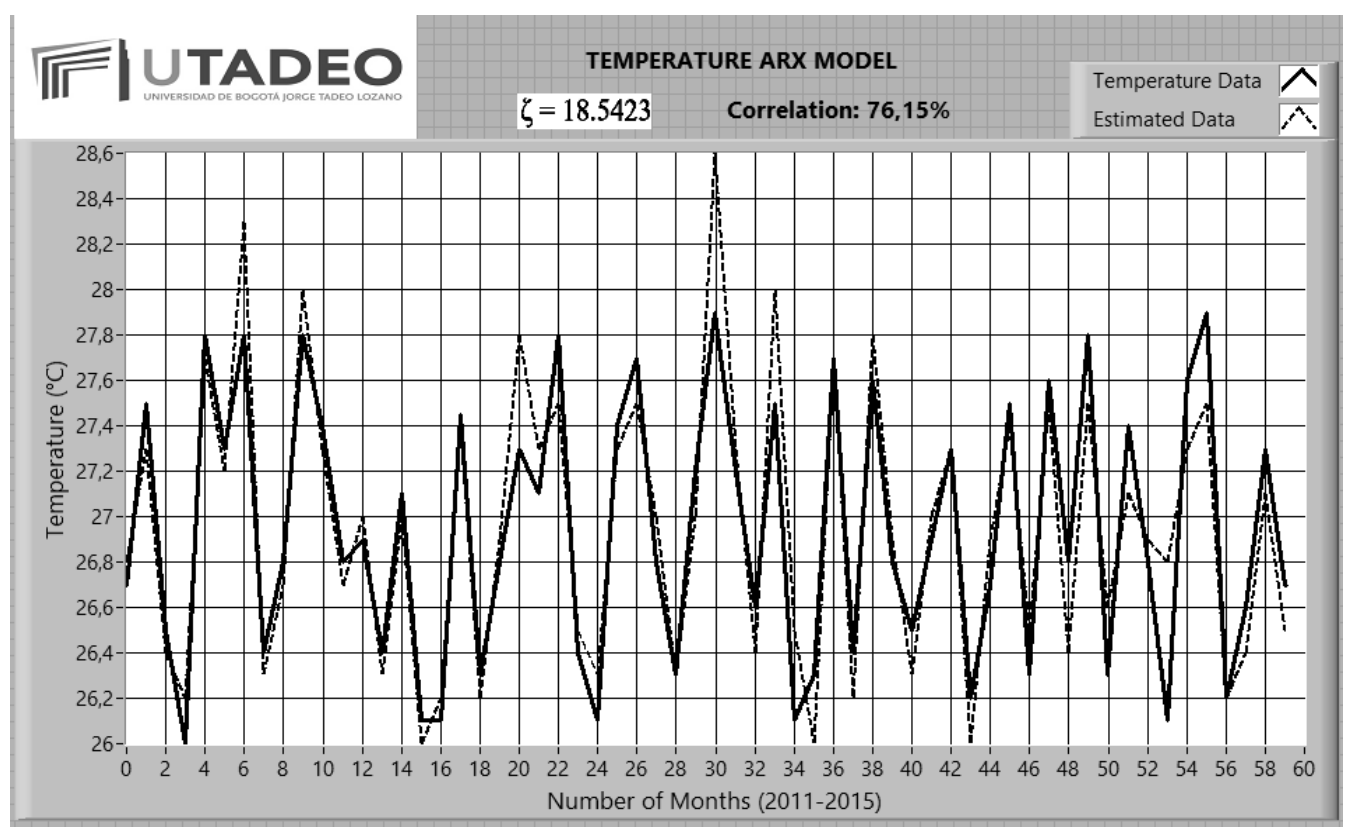

Fig. 3. Behavior for real temperature and the model estimated.

As these models need a regression vector of past data to calculate a new data, as for predicting or evaluating the model with the current data, it is necessary to know, at least, a number of data equal to the order of the model. The correlation obtained was calculated from the sample, in the same position of the order of the model, to the end of the data because just in this space it is possible to observe the behaviour of the model. This was also taken into account when drawing the graph and therefore, it is observed that the results of the graph are the same from the beginning until the data in the position of the order [24].

The results indicate the possible order of the resulting model that represents the data and the possible order of the real system. As the order increases, the program generates models unstable in their behaviour with respect to the real, causing the correlation to change significantly with respect to the previous model.

\section{Conclusion}

This paper presents a descriptive analysis of the variables of solar radiation and ambient temperature for the city of Bogotá. The results obtained show the typical characteristics for these tropical regions.

The application of the autoregressive model with exogenous input allows us to obtain a polynomial for the behaviour of the solar radiation with a correlation above $86 \%$, showing the applicability of the algorithm 
Virtual Instrumentation Applied to Identifying Parameters of Solar Radiation and Ambient..

implemented in LabVIEW. The model obtained for temperature was of order 12 with $\zeta=18.5423$, the correlation obtained was $76.15 \%$. The models applied as well as the behaviours and correlations obtained for the patterns of solar radiation and ambient temperature represent a reliable source of information that could be used to interpret the environmental variables.

\section{Acknowledgements}

This work was carried out with the financial support of the researching project 633-11-14 from Universidad de Bogotá Jorge Tadeo Lozano.

\section{References}

[1]. M.C. Di Vincenzo, D. Infield, Detailed PV array model for non-uniform irra- diance and its validation against experimental data, Sol. Energy 97 (2013) 314e331.

[2]. W. Teixeira da Costa, J. Farias Fardin, L. de Vilhena, B. Machado Neto, Domi- ngos Sa vio Lyrio Simonetti, Estimation of irradiance and temperature using photovoltaic modules, Sol. Energy 110 (2014) 132e138.

[3]. I. Visa, B. Burduhos, M. Neagoe, M. Moldovan, A. Duta, Comparative analysis of the infield response of five types of photovoltaic modules, Renewable Energy 95 (2016) 178:190.

[4]. T. Zhou, W. Sun. Study on maximum power point tracking of photovoltaic array in irregular shadow. Electrical Power and Energy Systems 66 (2015), 227-234.

[5]. Zhou L, Wu J, Li QH. Survey of maximum power point tracking techniques for photovoltaic array. High Voltage Eng 2008; 34(6):1145-54.

[6]. Koran A, LaBella T, Jih L. High efficiency photovoltaic source simulator with fast response time for solar power conditioning systems evaluation. IEEE Trans Power Electron 2014;29:1285-97.

[7]. G. Ciulla, V. Lo Brano, V. Di Dio, G. Cipriani, A comparison of different one- diode models for the representation of IeV characteristic of a PV cell, Renew. Sustain. Energy Rev. 32 (2014) 684e696.

[8]. J. Hernández W. Vallejo, G. Gordillo, Practical method for estimating the power and energy delilvered by photovoltaic modules operating under non-standard conditions, Prog. Photovolt: Res. Appl. 2013; 21:867-875.

[9]. A. Lopes, A. Araújo, A. Méndes, L. Andrade, A dye-sensitized solar cell model implementable in electrical circuit simulators, Solar Energy 122 (2015) 169-180.

[10]. Y. Xu, X. Kong, Y. Zeng, S. Tao, X. Xiao, A modeling method for photovoltaic cells using explicit equations and optimization algorithm, Electrical Power and Energy Systems, 59 (2014) 23-38.

[11]. F. Toledo, J. Blanes, Analytical and quasi-explicit four arbitrary point method for extraction of solar cell single-diode model parameters, Renewable Energy 92 (2016) 346-356

[12]. C. Ruschel, F. Gasparin, E. Costa, A. Krezinger, Assesment of pv modules shunt resistance dependence on solar irradiance, Solar Energy, 133 (2016) 35-43.

[13]. A. Rizzo, A. Cester, N. Wrachien, N. Lago, L. Torto, M. Barbato, J. Favaro, S. Gevorgyan, M. Corazza, F. Krebs, Characterization and modeling of organic (P3HT:PCBM) solar cells as a function of bias and illumination, Saolar Energy Materials \& Solar Cells 157 (2016) 337-345.

[14]. J. Ma, Z. Bi, T. Ting, S. Hao, W. Hao, Comparative performance on photovoltaic model parameter identification via bio-inspired algorithms, Solar Energy 132 (2016) 606-616.

[15]. A. Dolara, S, Leva, G. Manzolini, Comparison of different physical models for PV power output prediction, Solar Energy 119 (2015) 83-99.

[16]. D. Lan, M. Green, Generalised distributed model of a solar cell: lateral injection effects and impact on cell design and characterization, Solar Energy Materials \& Solar Cells 147 (2016) 108-114.

[17]. M. Catelani, L. Ciani, M. Kazimierczuk, A. Reatti, Matlab PV solar concentrator performance prediction based on triple junction solar cell model, Measurement 88 (2016) 310-317.

[18]. D. Cotfas, P. Cotfas, S. Kaplanis, Methods and techniques to determine the dynamic parameters of solar cells: Review, Renewable and Sustainable Energy Reviews 61 (2106) 213-221.

[19]. A. Hamache, N. Sengouga, A. Meftah, M. Henini, Modeling the effect of $1 \mathrm{MeV}$ electron irradiation on the performance of n+-p-p+ silicon space solar cells, Radiation Physics and Chemistry 123 (2016) 103-108.

[20]. S. Reynolds, V. Smirnov, Modelling performance of two - and four- terminal thin film silicon tandem solar cells under varying spectral conditions, Energy Procedia 84 (2015) 251-260.

[21]. L. Guo, Z. Meng, Y. Sun, L. Wang, Parameter identification and sensitivity analysis of solar cell models with cat swarm optimization algorithm, Energy Conversion and Management 108 (2016) 520-528

[22]. C. Chen, K. Yu, W. Du, W. Zhao, G. Liu, Parameters identification of solar cell models using generalized oppositional teaching learning based optimization, Energy 99 (2016) 170-180.

[23]. Ljung L. (2008). Perspectives on System Identification. Congreso IFAC.

[24]. A. Chica, F. Rey, J. Aristizábal. Application of autoregressive model with exogenous inputs to identify and analyse patterns of solar global radiation and ambient temperature. International Journal of Ambient Energy 33 (2012) 177-183.

[25]. Ljung L. (2008). Perspectives on System Identification. Congreso IFAC.

[26]. Kun-Shu Huang; Chi-Kuang Hwang; Kuo-Bin Lin; Chia-Wen Wu; , "Time series analysis during the releasing arrow stage," Machine Learning and Cybernetics, 2009 International Conference on , vol.6, no., pp.3300-3304, 12-15 July 2009.

[27]. Zweibel K. Harnessing solar power: the photovoltaic challenge. 1st ed. New York: Plenum Publishing; 1990.

[28]. Aristizábal J, Gordillo G. Performance and economic evaluation of the first grid - connected installation in Colombia over 4 years of continuous operation. International Journal of Sustainable Energy 2011; 30(1): 34-46.

[29]. Aristizábal J, Gordillo G. Performance Monitoring Results of the First Grid-Connected BIPV Installation in Colombia. Renewable Energy 2008; 33(11): 2475-2484.

[30]. Johnson G, Jennings R. LabVIEW Graphical Programming. McGraw-Hill: USA, 2006.

[31]. Bishop R. Learning with LabVIEW 8. Prentice Hall: USA, 2007. 